Stéphane Nowak

École Normale Supérieure de Lyon

\title{
TRADUIRE LA POÉSIE À L'ÉPREUVE DU PLURILINGUISME
}

\section{Translating poetry: into the experience of multilingualism}

\begin{abstract}
How to translate multilingual poets, who place the concern for orality at the heart of their work? Based on the poems of Agrafiotis and Costa Monteiro, the article formulates some hypotheses on the practices of transcreation, linguistic hybridization, and etymological concerns allowing neologisms to be authorized. It is thus a question of translating the performative dimension of the poem.
\end{abstract}

Keywords : contemporary poetry, experimental poetry, neologisms, plurilingualism, translation

\section{Tradurre poesia: sulle orme del plurilinguismo}

\section{Riassunto}

Come tradurre poeti plurilingui che porgono al centro del loro lavoro la preoccupazione per l'oralità? In base ai poemi di Agrafiotis e di Costa Monteiro, nell'articolo vengono formulate alcune ipotesi sulle pratiche di transcreazione, di ibridazioni linguistiche e di preoccupazioni etimologiche che permettono ai traduttori di usare neologismi. Si tratta dunque di tradurre la dimensione performativa del poema.

Parole chiave : neologismi, plurilinguismo, poesia contemporanea, poesia sperimentale, traduzione

La traduction de poètes contemporains plurilingues - qualifiés d'«expérimentaux » dans leur démarche - pose un certain nombre de problèmes spécifiques. Nous étudierons ici les cas du poète grec Démosthène Agrafiotis et du poète portugais Alfredo Costa Monteiro. Ces deux poètes ont pour point commun d'être plurilingues ou d'utiliser plusieurs langues dans leur œuvre (le grec, le japonais et l'anglais pour Agrafiotis ; l'espagnol, le portugais et le français pour Costa Monteiro). L'hypothèse de départ pour ces deux poètes est la suivante : leur travail sur le contemporain de la langue est à la croisée d'une interrogation sur les origines de l'écriture, avec un certain héritage des pratiques poétiques des 
avant-gardes (le lettrisme, la poésie visuelle et concrète), et un souci central de la lettre et de l'oralité (les deux poètes sont aussi performeurs et artistes sonores).

La question de l'«intraduisible » n'a cessé de hanter la traduction poétique, sans s'en contenter. Le risque étant que la trop grande quantité de sens contenue dans un vers puisse aboutir à une perte de sens. Lautre difficulté est que ces auteurs s'attachent particulièrement à la matérialité de la lettre, son corps, son signifiant et aussi, son aspect acoustique, sonore. Cela correspond au souci de lutter contre «l'oubli des lettres » qui serait présent dans des traductions exclusivement soumises à des questions sémantiques. Mais ici se pose spécifiquement la question de l'oubli du sonore, de la négligence de la musicalité.

Pour Agrafiotis comme pour Costa Monteiro, et comme de nombreux autres poètes classés dans ce que l'on nomme «poésie action » (selon le terme de Bernard Heidsieck qui entendait sortir le poème du livre et de la page) ou « poésie performance » ou poème en chair et en os (Julien Blaine pour qui le poème existe fondamentalement dans sa manifestation, bien davantage que dans sa trace écrite), l'écrit n'est qu'un des éléments d'un dispositif poétique plus global et général. Le poème est pour Agrafiois comme Costa Monteiro, le lieu de réflexion et de disposition d'éléments tactiques pour ralentir l'évidence du retour aux mots. Un retour à la matérialité de la lettre contre tout primat du sens ou du « fonctionnel ». L'enjeu n'est pas ici d'accélérer la lecture, mais de la ralentir. Il s'agit donc de préserver la force esthétique de la lettre. Et d’assumer les héritages les plus anciens des Phéniciens et des Grecs concernant l'évolution de l'alphabet avec l'héritage des avant-gardes (et pas seulement des lettristes), et la lecture in situ, ici et maintenant.

La question de la traduction des sonorités, de l'oralité et des sensations a été abordée de front par Jessica Stephens (Stephens, 2015). Mais elle est encore trop peu prise en compte. Voilà pourquoi se révèle l'intérêt d'une traduction comparée prenant davantage en compte le souci de la lettre et de l'oralité.

\section{Le rapport à la lettre et au sonore comme deux matériaux hétérogènes différenciés}

La question de «l'harmonie imitative $\gg$ (Paul Claudel) ou de « la musique des mots $\gg$ (Yves Bonnefoy) est une question récurrente de la production poétique du cratylisme selon laquelle les noms ont un lien direct, imitatif, avec leur signification. Le rôle du poète serait alors de retrouver cette harmonie de la langue.

Pour le traducteur, cela suppose d'accepter que le son fasse sens, ou du moins que le sens ne soit pas le critère unique ni central en matière de 
traduction poétique, en prenant en compte que le son vibratoire agit sur un plan physiologique en suscitant des sensations physiques.

Mallarmé l'écrivait déjà dans Crise de vers, 1897 :

Les langues imparfaites en cela que plusieurs, manque la suprême [...]. À côté d'ombre, opaque, ténèbres se fonce peu ; quelle déception, devant la perversité conférant à jour comme à nuit, contradictoirement, des timbres obscur ici, là clair. Le souhait d'un terme de splendeur brillant, ou qu'il s'éteigne, inverse; quant à des alternatives lumineuses simples - Seulement, sachons n'existerait pas le vers : lui, philosophiquement rémunère le défaut des langues, complément supérieur (Mallarmé, 1945 : 363-364).

Ce souci des connotations est pleinement à prendre en compte par le traducteur, même si une certaine subjectivé à expérimenter est inévitable pour la modéliser.

Démosthène Agrafiotis est un passionné de poésie et de photographie. Il fait ses études à Athènes à Polytechnique puis poursuit ses études en sociologie industrielle. Il subit l'arrivée de la dictature des colonels en Grèce. Il s'exile aux États-Unis en 1968, à l'université de Wisconsin en pleine effervescence politique et culturelle. Il vit ensuite à Paris où il suit de nombreux séminaires, dont ceux de Michel Foucault et de Lévi-Strauss. Il travaille alors sur «les risques technologiques comme élément culturel » et s'intéresse particulièrement à l'interdisciplinarité émergente : Fluxus, Cage, l'intermedia. Il s'intéresse aussi aux mutations technologiques de l'imprimé avec l'arrivée de l'offset. Cette mutation du médium permet l'émergence de nombreuses revues. À l'instar de Julien Blaine, à la fois poète et créateur de plusieurs revues, le poète devient éditeur. En 1973, il publie son premier livre à Athènes if you work with me. C'est une poésie structurée par la mathématique. Il crée aussi la revue Clinamen (« déviation $»$ en grec).

La poésie d'Agrafiotis vise à « chercher à établir des connexions, à provoquer une multiplicité d'espaces, de niveaux, de territoires, d'arrangements, d'intersections, de fissures $\gg$ (Agrafiotis, 1991 : 9). Mais il faut bien en revenir aux mots avec leurs arrangements ; leur signification détournée, désarticulée et, pour conclure, voici la définition qu’en donne le poète : « je peux définir les mots comme explosion du silence comme écho de l'attente lacune ouverte de l'action molécules d'avenir ou révélation du je dans la durée successive » (Agrafiotis, $1991: 9)$.

En français, il est traduit du grec par Claire Benedetti aux éditions de l'Attente en 2005. La ponctuation spécifique est conservée : pas de majuscule initiale, mais un point à la fin de chaque poème. Les transformations apportées au texte original passent logiquement par des substitutions, adjonctions, par périphrase ou par transposition suppression, mais tentent de conserver le souci 
de la lettre. Le premier poème est d'emblée délicat à traduire puisqu'il joue sur l'adjonction d'une lettre à chaque vers.

Le poète Costa Monteiro développe dans Anima un travail de poésie sonore dans une version plurilingue en français, espagnol et portugais. Ce qui pouvait paraitre comme un texte tourné vers l'intérieur se mue en une mélopée, un mot en initiant un autre pour former une nouvelle langue hybride. Une sorte de mélange entre une écriture blanche du fragment décontextualisé, intime et essentialiste avec une pratique formellement issue des avant-gardes. Car si le sujet peut paraitre universel - le mal-être nommé « mal à l'âme »- la livraison ne l'est pas : nulle nostalgie, plutôt une musicalité du télescopage des langues dans une partition. « Anima » se donne ainsi comme un anagramme, mais « âme » ne peut produire la même combinatoire. Ainsi, le sens étymologique d'« anima » est relié à « animé », ainsi préférable.

\section{Problèmes visuels de mise en page}

Le vers avance par expansion chez Agrafiotis dès le début du poème.

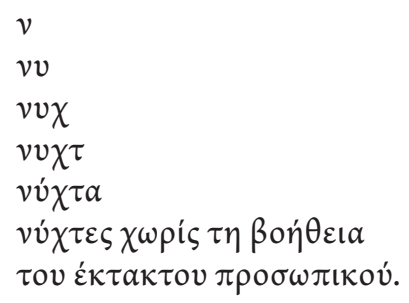

La traduction proposée conserve la mise en page et se lit ainsi :

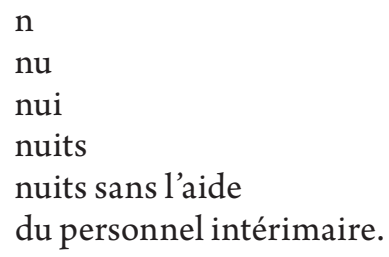

Pour le lecteur français, le choix d'《 intérimaire » conserve les connotations politiques du marché du travail précarisé.

Chez Costa Monteiro, le poème reproduit la forme de l'intestin grêle. Visuellement, le texte zigzague en serpent : il se lit donc de haut en bas, tantôt de droite à gauche, tantôt de gauche à droite à l'image de l'《iléon » (Costa Monteiro, 2015 : 34), troisième et dernière partie de l'intestin grêle. Par combinaison, mutation des lettres et des syllabes travaillant phonétiquement les $\ll 1 \gg, \ll \mathrm{m} \gg, \ll \mathrm{n} \gg$, les labiales et nasales, on passe vers l'anonyme, l'anormal, 
l'inhumanité, jusqu'à l'immolation et l'extinction, apocalyptique suicide. Le thème premier se dissémine donc en différentes directions beaucoup plus impersonnelles qu'on aurait pu le pressentir au départ. Il y a donc bien une ligne narrative souterraine vers le devenir animal, l'« anima animal $\gg$, l'« alimaña $\gg$, la bête monstrueuse. Se produit vers la fin un signal, un aigu sinusoïde perturbant et comme un dernier râle persistant. Une écriture de l'asphyxie dans un contexte de fin du monde. Lecture sèche avec des effets de réverbération des mots par l'intérieur, système d'échos. Hypnotique. Nous sommes donc en présence d'une forme de « performance typographique » selon Sébastien Dulude (cfr. Dulude, 2015).

La combinatoire sonore $\mathrm{d}^{\prime} \ll$ anima $\gg$ dérive ainsi par inversion de $\ll \mathrm{n} \gg$ et $\mathrm{du} \ll \mathrm{m} \gg$ dans $\ll$ à miner $\gg$.

\section{Racines, hybridation et dimension performative}

Il y a ainsi dans les traductions en français une forme d'hellénisation du français chez Agrafiotis et d'hispanisation chez Costa Monteiro.

Travailler les racines étymologiques permet ainsi de produire des néologismes compréhensibles par le lecteur français.

Le titre « N, al » est traduit en « Ou, i $»$. Il y a donc déplacement de la virgule interne au mot. Ce qui permet de lire $\langle$ ou $\gg /$ 《oui $\gg$ (ce qui n'est pas dans le texte original). Le i reste de côté, mails il ne s'entend pas en grec.

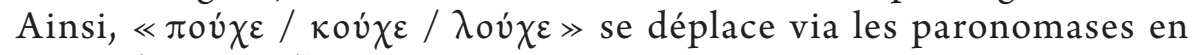
«poumain/coumain/loumain ».

Chez Costa Monteiro le mot «âme » est hybridé avec « animé »se qui permet de jouer avec l'étymologie (anima), une transcréation d'emblée à l'œuvre.

Le problème des transpositions musicales est lié à la difficulté d'en mesurer l'effet sans expérimentation, par la lecture orale justement.

Le problème est alors d'évaluer l'effet et l'impact de la traduction sur le lecteur. Cela suppose à la fois une pratique d'expérimentation et une réflexion pragmatique. Il est en effet indispensable d'évaluer une certaine dimension performative du langage en rapport justement avec des poètes dits de la performance.

Pour Agrafiotis, la performance est « epitelesis », en train de se finaliser, une certaine définition de la traduction par le mouvement, le processus, davantage que par le résultat.

Ainsi, ces problèmes de transcréations poétiques remettent la question de la lettre au cœur du souci du traducteur (Berman, 1999) pour tenter une traduction performative. 


\section{Bibliographie}

Agrafiotis D. (1991) : Traduction E. Hocquard “Déviations”. Paris : Les Cahiers de Royaumont.

Agrafiotis, D. (2005) : Ou,i. Bordeaux : éditions de L'attente.

Agrafiotis, D. (2016) : Y,es \& Diaeresis. Kingston : Dusie.

Berman, A. (1999) : La traduction et la lettre ou l'auberge du lointain. Paris : Seuil.

Costa Monteiro, A. (2015) : Anima (coffret+CD). Lenka lente, presses du réel.

Dulude, S. (2015) : Performativité des dispositifs typographiques du livre de poésie de contre-culture québécoise : regards culturels et littéraires. Trois-Rivières : Université du Québec à Trois-Rivières. En ligne : depot-e.uqtr.ca/7675/1/031014346.pdf (consulté le 7.12.2018).

Mallarmé, S. (1945) : Variations sur un sujet. In : Mallarmé, S., Euvres Complètes, pp. 363-364. Paris : Gallimard, Bibliothèque de la Pléiade.

Stephens, J. (2015) : Présentation. Palimpsestes, 28, 2015, pp. 11-22, http://journals. openedition.org/palimpsestes/2246 (mis en ligne le 1.11.2015, consulté le 7.12.2018). DOI : https://doi.org/10.4000/palimpsestes. 2246 